\title{
Thermal degradation of natural and treated hemp hurds under air and nitrogen atmosphere
}

\author{
Nadezda Stevulova $^{1}$ (D) Adriana Estokova $^{1} \cdot$ Julia Cigasova $^{1} \cdot$ Ivana Schwarzova $^{1} \cdot$ \\ Frantisek Kacik ${ }^{2}$ - Anton Geffert ${ }^{2}$
}

Received: 21 July 2016/ Accepted: 8 December 2016/Published online: 27 December 2016

(C) The Author(s) 2016. This article is published with open access at Springerlink.com

\begin{abstract}
Sustainability goals are essential driving principles for the development of innovative materials in the construction industry. Natural fibers represent an attractive alternative as reinforcing material due to good mechanical properties and sustainability prerequisites. The study has been focused on the comparative investigation of chemical and physical treatments of hemp hurds and their influence on the thermal behavior of main hemp constituents in air and nitrogen atmosphere. Thermal decomposition of hemp hurds involves several parallel reactions related to heat and mass transfer processes. A comparison of DSC and TG/ DTG results of hemp hurds samples before and after treatments demonstrates a better thermal stability for treated samples. It is caused by changes in chemical composition due to a partial removal of non-cellulosic components from hemp hurds structure, an increase in cellulose content and decrease in its degree of polymerization. The results show different thermal behavior of the hurds samples heated under nitrogen and air atmosphere. Based on DTG records, several-stage process of mass loss has been found for the samples under air, whereas only two-stage process under nitrogen.
\end{abstract}

Nadezda Stevulova

nadezda.stevulova@tuke.sk

1 Department of Material Engineering/Institute of Environmental Engineering, Faculty of Civil Engineering, Technical University of Kosice, Vysokoskolska 4, 04200 Kosice, Slovakia

2 Department of Chemistry and Chemical Technologies, Faculty of Wood Sciences and Technology, Technical University in Zvolen, T. G. Masaryka 2117/24, 96053 Zvolen, Slovakia
Keywords Degree of polymerization - Hemp hurds . Chemical composition - Treatment processes - Thermal degradation - Thermal stability

\section{Introduction}

The increasing global energy consumption, economic crisis and environmental risks have encouraged research on the exploitation of renewable raw materials resources for production of environmentally friendly materials. Recently, the hotspot interest in the use of rapidly renewable raw materials resources such as vegetable fibers with high tensile strength as a good reinforcing component and/or organic filler into composite materials for automotive [1] and building sector $[2,3]$ is recorded. For utilization of natural materials in buildings, their thermal stability has to be taken into account. Thermal analysis can help in understanding the degradation mechanism as well as in enhancement the thermal stability of a polymeric material. Composites based on biomass usually have better thermal properties than corresponding components in the system [4].

Changes in the structure of biomass during thermal treatment have been studied in several studies [5-8]. Extensive research has showed the complexity of thermal decomposition reactions of natural fibers due to the complex structure of the lignocellulosic material representing heterogeneous mixture of organic compounds [9]. The mass loss of the main components of biomass occurs in a determined temperature range depending on experimental conditions and biomass type. Different reaction schemes and approaches have also been considered for a better interpretation of biomass degradation process [10]. 
One of the potential composite reinforcing materials, industrial hemp (Cannabis sativa L.), is considered. This plant is one of the world's oldest cultivated and fastest growing annual fiber plant delivering fibers, hurds and seed. This crop has a very high yield of biomass and therefore is suitable for wide range of industrial uses. Because of its unique properties (thermal insulation, antiseptic, acoustic and mechanical), particularly its environmental benefits and low content of tetrahydro cannabinoids (THC), hemp is considered a valuable crop for the bio-based economy [11]. The integrated use of biopolymer components of hemp is very important for the biorefining industry [12].

The technical hemp is the source of two types of fibers: bast fibers (non-wood fibers) from the outer portion of the stem and woody core fibers, called shives or hurds from the interior part of the stem. The hurds represent about $60-80 \%$ of the stem in hemp. Each produced kilogram of hemp fiber gets as a by-product $1.7 \mathrm{~kg}$ of hemp hurds [13]. The hurdsto-fibers ratio varies from 1.5 to more than 2 [14]. Hemp hurds as solid waste biomaterial of fiber production consist of three major polymeric components (cellulose, hemicelluloses and lignin). Water-soluble substances, pectin and waxes existing in water insoluble forms are present in low contents. Amorphous components such as hemicelluloses, pectin and lignin have a low thermal stability and tend to degradation at relatively low temperatures (below $500{ }^{\circ} \mathrm{C}$ ) $[15,16]$. The changes in hemp fiber/hurds during heating were investigated in some papers [5, 17-19]. Temperature range of hemp hurds degradation depends on chemical composition of biomass and conditions of heating [20]. The physical changes are related to enthalpy, mass, color, strength, crystallinity and orientation of microfibril angle [21]. Thermal degradation of hemp hurds components is connected with chemical changes occurring at the molecular level of the polymer constituents as a function of progressive temperature. Hemp polymer constituents decompose into substances with shorter chain length and different molecular mass distribution. Thermal behavior of hemp hurds described in [22] is similar to hemp fiber compared to the initial degradation temperature $\left(150-200{ }^{\circ} \mathrm{C}\right)$ [23]. Heating of the hemp fibers in the temperature range of 160 and $260{ }^{\circ} \mathrm{C}$ has resulted in softening of lignin leading to opening of fiber bundles into individual fibers [24]. The effect was more pronounced for fibers heated in air than for those in inert (nitrogen) environment.

Pre-treatment of vegetable fibers for their practical use into biocomposites is an important tool in order to remove impurities and amorphous components, increase accessible surface area and reduce hydrophilic nature of biomass constituents. To address this challenge, natural fibers are subjected to physical, chemical or biological methods of surface modification, but some procedures incorporate the combination of two methods. In terms of simplicity and creating favorable conditions for the formation of bonds between the fiber and matrix particles, chemical modification of fibers has been used more frequently. The treatment effects on the thermal decomposition of hemp components have been studied in some papers. The untreated, hot water washed and alkali-treated hemp samples were compared in terms of thermal properties [25]. The contribution of the main components in the structure of hemp fibers chemically treated by three different agents to their thermal properties was studied by using TG/DTA analysis under inert atmosphere (argon) [26]. Thermal stability of treated samples decreased in the following order: $\mathrm{NaOH}$-treated fibers, silane-treated fibers, solvent extracted fibers and untreated hemp fibers. According to detail TG and DSC analysis of alkalized, acetylated and silanized hemp fibers and individual hemp components (cellulose, hemicelluloses and lignin) in work [27], the treated fibers have higher thermal stability compared to the untreated fibers. The most reactive constituent is hemicelluloses, lignin decomposes in a wide temperature range and cellulose is more thermally stabile. While explaining degradation of the main components in hemp fibers has been paid, decomposition of hemp hurds constituents during heating has not been sufficiently clarified. Therefore, the main objective of this study has been focused on the comparative investigation of influence of chemical, physical treatment and combination of both methods of hemp hurds on the thermal decomposition of its constituents in air and nitrogen atmosphere.

\section{Materials and methods}

Industrial hemp hurds coming from the Netherlands Company Hempflax with a wide particle size distribution (8-0.063 $\mathrm{mm})$, analyzed by mechanical sieving, are used in experiment. Critical parameter of particles dimension is length, since the width and thickness of hemp hurds varied only slightly. The mean particle length of hurds slices (1.94 mm) was calculated as the first moment of the density of the mass size distribution function. Reproducibility of particle size measurement ranged about $5 \%$. The used hemp hurds contains the small amount of bast fibers. Density of hemp material was $117.5 \mathrm{~kg} \mathrm{~m}^{-3}$. The average moisture content of the raw hemp material was found out 10.78 mass $\%$.

\section{Treatment procedures}

Prior to treatment and in order to ensure constant humidity content, fibers were dried at $80{ }^{\circ} \mathrm{C}$ for $24 \mathrm{~h}$ in a drying 
oven. The dried samples were stored in desiccators with a sealed plastic bag to avoid atmospheric moisture contamination. The dried hemp hurds were treated by chemical and physico-chemical methods. After treatment, removing any solution residues of chemicals used for treatment of hemp hurds follows. All samples were continuously washed with deionized water until the $\mathrm{pH}=7$. Alkalitreated sample after washing was neutralized in a $1 \mathrm{vol} \%$ acetic acid. Subsequently, all samples were dried in an oven at $80{ }^{\circ} \mathrm{C}$ until a constant mass was reached. Constant mass has been achieved when less than $0.1 \%$ of the test sample wet mass was lost during an additional exposure to the drying process.

\section{Chemical treatment}

In this study, three different chemical agents for surface modification of dried hemp hurds slices were used: water solutions of ethylenediaminetetraacetic acid (EDTA; Gavax, Slovakia), calcium hydroxide (Roth, Germany) and sodium hydroxide (Chemapol, Slovakia). Treatment experiments were carried out at room temperature under conditions given in [28]. Treatment by EDTA was made by $3 \mathrm{~h}$ immersion of hemp hurds sample in a saturated solution of EDTA $\left(5 \mathrm{~g} \mathrm{l}^{-1}\right)$. In order to saturate fibers with $\mathrm{Ca}$ +2 ions, sample was placed in a saturated lime solution $\left([\mathrm{Ca}+2]=2.10^{-2} \mathrm{M}\right)$ for 48 h. $1.6 \mathrm{M} \mathrm{NaOH}$ solution was used for alkalization of hemp material (48 h).

\section{Physico-chemical treatment}

Three ways of physico-chemical pre-treatment of hemp hurds as ultrasonic treatment in distilled water and in $\mathrm{NaOH}$ solution and hot water treatment were used. An ultrasonic bath TESON 10 (Tesla, Slovakia) was used for ultrasonic cleaning process of organic material. This device of $10 \mathrm{~L}$ tub capacity is mono-frequency with low voltage $(220 \mathrm{~V}, 50 \mathrm{~Hz}, 650 \mathrm{~W}$ of power output). The experiment was performed in distilled water and $0.2 \mathrm{M} \mathrm{NaOH}$ solution at solid to liquid ratio of 1:10 during $60 \mathrm{~min}$ sonication. Hot water washing treatment of hemp hurds was realized by its cooking in boiling distilled water $\left(100^{\circ} \mathrm{C}\right)$ for 60 min under the same value of s/l ratio.

The nomenclature and abbreviation used for the hemp hurds samples are in Table 1.

\section{Methods of chemical analysis}

A milled (under $40.10^{-3} \mathrm{~mm}$ ) and oven-dried sample was used for the determination of chemical composition of hemp hurds. Methods of hemp components determination as following: Extractives were determined in a Soxhlet apparatus with a mixture of ethanol and toluene (2:1) for
Table 1 Denoting of used hemp hurds samples

\begin{tabular}{ll}
\hline Hemp hurds samples & Abbreviation \\
\hline Reference (original) & RHH \\
$\mathrm{NaOH}$ treated & ATHH \\
$\mathrm{Ca}(\mathrm{OH})_{2}$ treated & CTHH \\
EDTA treated & ETHH \\
Hot water boiled & HWTHH \\
Ultrasound treated in water & UWTHH \\
Ultrasound treated in $\mathrm{NaOH}$ & UATHH \\
\hline
\end{tabular}

$8 \mathrm{~h}$ according to the ASTM Standard D 1107-96 (1998). Total content of polysaccharides (i.e., holocellulose) was determined using the method of Wise et al. [29]. Cellulose content was determined by the Seifert method [30]. The content of hemicelluloses was determined as the difference between holocellulose and cellulose. The content of acidinsoluble (Klason) lignin was determined according to NREL procedure [31]. The samples were hydrolyzed in a two-stage process. In the first stage, $72 \%$ (w/w) $\mathrm{H}_{2} \mathrm{SO}_{4}$ at a temperature of $30{ }^{\circ} \mathrm{C}$ was used for $2 \mathrm{~h}$, and in the second stage, the samples were refluxed after dilution to $4 \%(\mathrm{w} / \mathrm{w})$ $\mathrm{H}_{2} \mathrm{SO}_{4}$ for $4 \mathrm{~h}$. Total ash content (mineral substances) was determined according to NREL procedure [32].

\section{Size exclusion chromatography}

Molecular mass distribution analysis of the cellulose samples was performed by size exclusion chromatography (SEC) after their conversion into tricarbanilates according to modified procedure [33]. Briefly, the cellulose samples were dried over silica gel for several days. Anhydrous pyridine $(8.0 \mathrm{~mL})$, cellulose $(50 \mathrm{mg})$ and phenyl isocyanate $(1.0 \mathrm{~mL})$ were sealed in a $50 \mathrm{~mL}$ dropping flask and heated in an oil bath at $70{ }^{\circ} \mathrm{C}$ for $72 \mathrm{~h}$. At the end of the reaction, methanol $(2.0 \mathrm{~mL})$ was added to the mixture to eliminate the excess of phenyl isocyanate. The yellow solutions then were added dropwise into a rapidly magnetic stirring 7:3 methanol/water mixture $(150 \mathrm{~mL})$. The solids were collected by filtration and washed with 7:3 methanol/ water mixtures $(1 \times 50 \mathrm{~mL})$ followed by water $(2 \times 50 \mathrm{~mL})$ to neutral reaction. The cellulose tricarbanilate was air dried overnight, then under vacuum at $50{ }^{\circ} \mathrm{C}$. Cellulose tricarbanilates were dissolved in tetrahydrofuran and filtered through a Puradisc 25 NYL filter (Whatman International, Maidstone, UK) with a pore size of $0.45 \cdot 10^{-3} \mathrm{~mm}$. SEC was performed at $35^{\circ} \mathrm{C}$ with tetrahydrofuran at a flow rate of $1 \mathrm{~mL} \mathrm{~min}^{-1}$ on two PL gel (porous polystyrene/divinylbenzene matrix with particle size of $10 \mu \mathrm{m}$ and internal diameter $\times$ length of $7.5 \times 300 \mathrm{~mm}$ ) MIXED-B columns (Agilent Technologies, Santa Clara, CA, USA) preceded by a PL gel 
$\left(10.10^{-3} \mathrm{~mm}, \quad 7.5 \times 50 \mathrm{~mm}\right), \quad$ Guard-column (Agilent Technologies) as described by Kacik et al. [33]. Data acquisitions were carried out with ChemStation software (Agilent Technologies), and calculations were performed with the Clarity GPC (Gel Permeation Chromatography) module (DataApex, Prague, Czech Republic). Numerical outputs obtained for Mn (number-average molecular mass) and $\mathrm{Mw}$ (mass-average molecular mass) were recalculated to underivatized cellulose by multiplication with the coefficient $k=162 / 519$. Polydispersity index (PDI) of cellulose was calculated as the ratio Mw/Mn. Degree of polymerization (DP) values were calculated by dividing the molecular mass by the monomer equivalent mass of anhydroglucose $(\mathrm{DPw}=\mathrm{M} / 162)$.

\section{Thermo-gravimetric and differential scanning calorimetric analysis}

Dynamic experiments were performed using a model STA 449F3 (Netzsch, Germany) of simultaneous TG/DSC system. Thermal decomposition of the raw hemp hurds and treated samples was monitored in the standard temperature range from room temperature to $900{ }^{\circ} \mathrm{C}$ under air and inert atmosphere (nitrogen), at a heating rate of $10{ }^{\circ} \mathrm{C} \mathrm{min}^{-1}$. Alumina crucible $\left(\mathrm{Al}_{2} \mathrm{O}_{3}\right)$ was used in DSC/TG measurements. The sample amount was $10 \pm 2 \mathrm{mg}$ per specimen for thermo-gravimetric (TG) and differential scanning calorimetric analysis (DSC).

\section{Results and discussion}

\section{Effect of hemp hurds treatments on chemical composition}

Changes in chemical composition of chemically and physico-chemically treated hemp hurds in comparison with the original sample are shown in Table 2. By comparing the contents of main components of hemp hurds samples, some interesting observations have been found. According to the literature data [34], physico-chemical treatment did not lead to significant changes in content of the main hemp hurds components. No change in mass of hemp hurds samples UWTHH and HWTHH was observed. But ultrasound treatment of hemp hurds in $\mathrm{NaOH}$ solution (UATHH sample) caused a reduction in hemicelluloses and holocellulose contents about 28 and $9 \%$, respectively. On the other hand, differences between contents of hemp hurds components in chemically modified samples compared to original sample were observed. The most significant decrease in contents of hemicelluloses, cellulose and lignin was recorded for sample modified in $\mathrm{NaOH}$ solution. Decrease in polysaccharides component (holocellulose) is caused mainly by degradation of hemicelluloses. This change is accompanied by an increase in the cellulose and lignin contents. It is generally known that alkali $(\mathrm{NaOH})$ treatment reduces the number of hydroxyl groups of the amorphous materials cementing hemp hurds, destruct the cellular structure and thereby hydrophobic nature of hemp hurds increases. As shown in Table 2, the contents of the main hemp components in samples modified by using EDTA and $\mathrm{Ca}(\mathrm{OH})_{2}$ did not exhibit differences in comparison with original hemp hurds. Some differences in reduction of lipophilic extractive compounds and ash in all treated samples in comparison with original sample were observed as well. The lower content of ash in treated samples comparing to the untreated sample can result of changes in chemical composition due to treating or washing. RHH sample was not treated in any way.

\section{Changes in polydispersity and degree of cellulose polymerization}

The values of number-average molecular mass (Mn), massaverage molecular mass $(\mathrm{Mw})$ and polydispersity index (PDI) of cellulose in reference sample and treated hemp hurds are reported in Table 3. As it can be seen, the differences in the values of Mw and PDI were more significant than in case of parameters Mn with the exception of UATHH sample. The most significant changes in Mw and

Table 2 Comparison of changes in hemp hurds components content before and after treatment

\begin{tabular}{llccccc}
\hline Main components of hemp hurds/\% & \multicolumn{3}{l}{ Hemp hurds samples } & & & \\
\cline { 2 - 7 } & RHH & ATHH & CTHH & ETHH & HWTHH UWTHH & UATHH \\
\hline Holocellulose & 77.3 & 66.0 & 74.5 & 76.7 & 77.277 .8 & 68.8 \\
Cellulose & 44.5 & 53.9 & 45.8 & 45.7 & 45.846 .7 & 45.1 \\
Hemicelluloses & 32.8 & 12.1 & 28.7 & 31.0 & 32.332 .6 & 23.7 \\
Lignin & 22.0 & 27.4 & 24.0 & 24.2 & 21.523 .2 & 24.1 \\
Compounds soluble in toluene and ethanol & 3.5 & 2.8 & 2.5 & 2.9 & 2.42 .6 & 4.1 \\
Ash & 2.6 & 1.3 & 1.5 & 1.1 & 1.1 & 1.3 \\
\hline
\end{tabular}


Table 3 Values of number-average molecular mass (Mn), mass-average molecular mass (Mw), polydispersity index (PDI) of cellulose and degree of polymerization of cellulose (DP) in reference sample and treated hemp hurds

\begin{tabular}{lrrrr}
\hline Hemp hurds sample & Mw $\times 10^{3}$ & Mn $\times 10^{3}$ & PDI & DP $\times 10^{3}$ \\
\hline RHH & $210.9 \pm 6.4$ & $18.6 \pm 0.6$ & $11.3 \pm 0.85$ & $1.3 \pm 0.04$ \\
ATHH & $94.9 \pm 1.2$ & $15.5 \pm 0.7$ & $6.1 \pm 0.20$ & $0.6 \pm 0.07$ \\
CTHH & $141.2 \pm 6.1$ & $15.1 \pm 0.7$ & $9.3 \pm 0.07$ & $0.9 \pm 0.04$ \\
ETHH & $150.4 \pm 6.7$ & $14.7 \pm 0.6$ & $10.2 \pm 0.12$ & $0.9 \pm 0.04$ \\
HWTHH & $183.7 \pm 4.1$ & $15.6 \pm 0.8$ & $1,181 \pm 0.30$ & $1.1 \pm 0.03$ \\
UWTHH & $147.4 \pm 3.4$ & $13.1 \pm 0.5$ & $11.2 \pm 0.14$ & $0.9 \pm 0.02$ \\
UATHH & $27.9 \pm 0.9$ & $5.9 \pm 0.2$ & $4.7 \pm 0.04$ & $0.2 \pm 0.01$ \\
\hline
\end{tabular}

PDI values were in cellulose of hemp hurds sample treated by ultrasound (UATHH) and chemical method in $\mathrm{NaOH}$ solution (ATHH). Mw value of ATHH sample reached only $45 \%$ of value measured for reference sample (RHH), but this value for sample UATHH decreased up to the level of $13 \%$ of RHH. The value of PDI of cellulose in hemp hurds samples decreased from $11.33(\mathrm{RHH})$ to 6.13 (ATHH) and 4.69 (UATHH).

Decrease in polydispersity was observed in chemically, irradiated and enzymatically treated celluloses [35]. This fact is connected with the degradation of high molecular mass fractions into cellulose chain of shorter lengths. The PDI value of ultrasound sample (UWTHH) is almost the same as the RHH. Hot water treatment (HWTHH) led to the increase of PDI by $90 \%$ when compared to RHH. An increase in polydispersity during cotton fibers aging at higher temperatures $\left(120-160^{\circ} \mathrm{C}\right)$ was found [36].

The important structural parameter of cellulose is its degree of polymerization (DP). According to [37], the DP can be used as a measure of cellulose degradation, caused mainly by physical or chemical influences or by irradiation. The studied treatment procedures caused reduction in DP values of cellulose. Decrease in DP relates to the cleavage of glycosidic bonds and shortening cellulose chains. The most significant decrease in DP value for the hemp hurds sample UATHH (87\%) in comparison with RHH was observed. It seems that this fact relates to ultrasound process mechanism.

When ultrasound passes through a liquid medium, the interaction among ultrasonic waves, liquid and dissolved gas leads to acoustic cavitation that may affect morphology and surface of material. A cavitation as most striking property of ultrasound is responsible for suddenly collapse of cavities, creating power shock waves and generating a large amount of mechanical and thermal energy in the liquid [38]. Local high temperature and pressure placed in volume of liquid arise, and consequently the complex structure of bundles of fibers and fibrils is broken. Ultrasound treatment can enhance existing extraction processes and enable new commercial extraction opportunities and processes [39].

\section{Thermal degradation of hemp hurds components}

In Figs. 1 and 2, DSC, TG and DTG curves of untreated and treated hemp hurds samples in nitrogen and air atmosphere are presented.

The maximum temperature and temperature ranges of hemp hurds components decomposition and the corresponding mass loss for studied samples under both atmospheres are given in Table 4. The DSC curves indicate three temperatures effects in which the mass loss of the

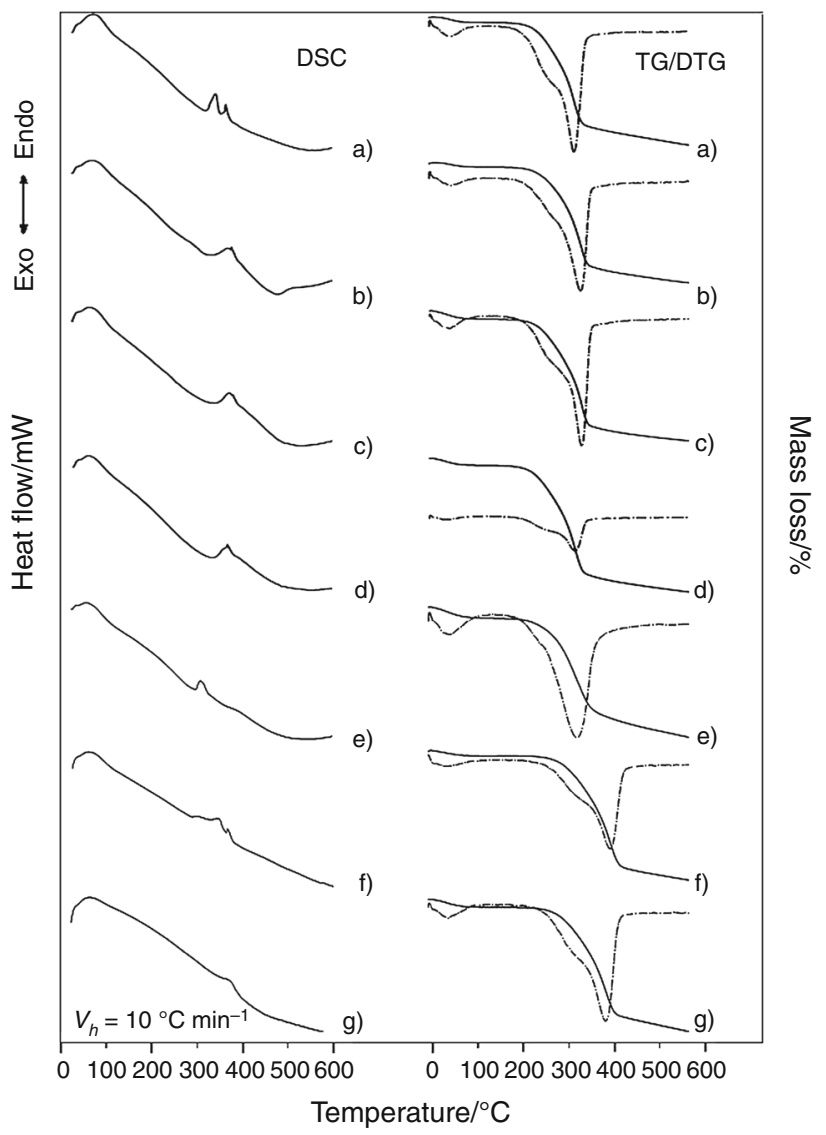

Fig. 1 DSC and TG/DTG curves in nitrogen atmosphere of reference hemp hurds and treated samples ( $a \mathrm{RHH} ; b \mathrm{NTHH} ; c \mathrm{CTHH}$; $d$ ETHH; $e$ UATHH; $f$ UWTHH; $g$ HWTHH) 


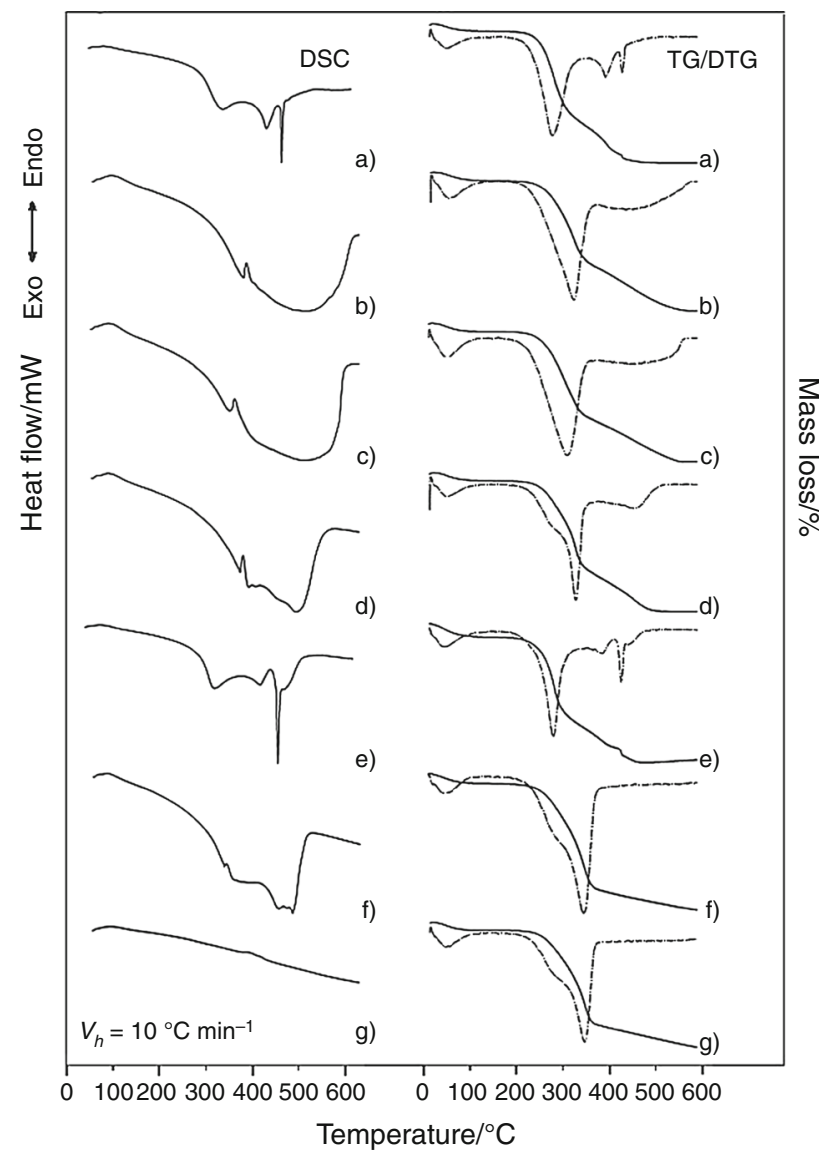

Fig. 2 DSC and TG/DTG curves in air atmosphere of reference hemp hurds and treated samples ( $a \mathrm{RHH} ; b \mathrm{NTHH} ; c \mathrm{CTHH}$; $d$ ETHH; $e$ UATHH; $f$ UWTHH; $g$ HWTHH)

organic compounds occur. Three steps of thermal degradation of hemp hurds samples related to hemicelluloses, cellulose and lignin are observed. The distinction between the main hurds components is possible by means of DTG curves for all samples under nitrogen atmosphere; however, such a distinction is not possible for the RHH, NTHH and CTHH samples heated under air atmosphere due to overlapping of hemicelluloses, cellulose and lignin peaks during the heating process.

\section{Referential hemp hurds (RHH)}

DSC curves of natural hemp fibers (Figs. 1a, 2a) obtained by analysis in nitrogen and air atmosphere show an initial endothermic peak between 50 and $100{ }^{\circ} \mathrm{C}$. The initial 3-4\% of mass loss (air and nitrogen atmosphere) is attributed to the evaporation of inherent air moisture and physically adsorbed water in hurds [18]. The DSC curve (air) showed other three exothermic peaks centered around 312,395 , and $437{ }^{\circ} \mathrm{C}$, whereas in nitrogen atmosphere is thermal decomposition accompanied by three endothermic processes (peaks around
320-335, 358 and $311-384{ }^{\circ} \mathrm{C}$ ). Atmosphere change can cause the change in nature of chemical degradation process as reported in [26]. Cellulose decomposition is generally endothermic process in inert atmosphere. Under nitrogen atmosphere, the cellulose is more resistant against thermal treatment $[40,41]$. This is likely due to a crystalline structure of cellulose. When under air heated, cellulose ongoing three primary reactions: thermo-oxidation, dehydration and depolymerization resulting in glycosane formation occur in the temperature range of $120-350{ }^{\circ} \mathrm{C}$ [42]. Thermo-oxidation and dehydration are driven by diffusion processes and run mainly in amorphous phases of polysaccharides. Simultaneously, a depolymerization of cellulose is observed and leads to a decrease in an average polymerization degree. Heating of cellulose under air atmosphere causes oxidation of hydroxyl groups in cellulose molecules and number of carbonyl groups increase. Subsequently, carboxylate compounds are formed. At temperatures above $300{ }^{\circ} \mathrm{C}$, depolymerization becomes the dominant reaction of the degradation of cellulose.

Depolymerization of hemicelluloses and pectin run simultaneously. According to [26], where thermal analysis of extracted components of hemp fibers was investigated, pectins are degraded at lower temperature (about $280{ }^{\circ} \mathrm{C}$ ). The peak position is depending on the heating rate [43]. Difference in the maximum temperature of pectin's decomposition depends on the mass loss rate as well as on nature of atmosphere.

Exothermic peaks for depolymerization of hemicelluloses are observed around $320-335{ }^{\circ} \mathrm{C}$ and near $312{ }^{\circ} \mathrm{C}$ in nitrogen and air atmosphere, respectively. As reported in [44], exothermic decomposition of hemicelluloses occurs in the temperature range of $220-315^{\circ} \mathrm{C}$. According to [27], the majority of the hemicelluloses decomposed in the temperature range of $180-200{ }^{\circ} \mathrm{C}$. The endothermic decomposition of pectin may be largely counter balanced by the exothermic depolymerization of hemicelluloses [26]. As shown in [45], in temperature range of $220-375^{\circ} \mathrm{C}$, lignin is partially decomposed. The determined mass loss for these temperature range corresponding two peaks was about $59.7 \%$ in air and $24.9 \%$ in nitrogen atmosphere. The endothermic peak observed on DSC curve (nitrogen) at $358{ }^{\circ} \mathrm{C}$ of RHH sample contains a contribution due to the cellulose decomposition. This peak is shifted to $395{ }^{\circ} \mathrm{C}$ (mass loss $23.9 \%$ ) in air atmosphere. In fact, at this temperature, the decomposition rate (DTG) in air is higher than that observed in nitrogen atmosphere (Figs. 1a, 2a). These findings on the hemicelluloses and cellulose decomposition are in good agreement with the literature data [18].

The sum of the mass losses achieved after hemp hurds components decomposition in this temperature range in air corresponds to $83.6 \%$. This value is higher than content of 
Table 4 Temperature ranges of hemp hurds components degradation and corresponding mass losses (nitrogen and air atmosphere)

\begin{tabular}{|c|c|c|c|c|c|}
\hline \multirow[t]{2}{*}{ Treatment } & \multirow[t]{2}{*}{ Sample } & \multicolumn{4}{|c|}{ Temperature ranges of components degradation $/{ }^{\circ} \mathrm{C}$ under nitrogen and air atmospher } \\
\hline & & 1st peak & 2nd peak & 3rd peak & Total mass loss $/ \%$ \\
\hline \multirow[t]{4}{*}{ Chemical (nitrogen atm.) } & RHH & $320-335^{\circ} \mathrm{C}$ & $358^{\circ} \mathrm{C}$ & $311-384{ }^{\circ} \mathrm{C}$ & 79.10 \\
\hline & ATHH & $320-370{ }^{\circ} \mathrm{C}$ & $371{ }^{\circ} \mathrm{C}$ & $320-385^{\circ} \mathrm{C}$ & 78.98 \\
\hline & ETHH & $330-358{ }^{\circ} \mathrm{C}$ & $364{ }^{\circ} \mathrm{C}$ & $330-390{ }^{\circ} \mathrm{C}$ & 79.20 \\
\hline & CTHH & $337-377^{\circ} \mathrm{C}$ & $378{ }^{\circ} \mathrm{C}$ & $349-390{ }^{\circ} \mathrm{C}$ & 81.81 \\
\hline \multirow[t]{3}{*}{ Physico-chemical (nitrogen atm.) } & UWTHH & $207-382{ }^{\circ} \mathrm{C}$ & & - & 84.34 \\
\hline & UATHH & $178-347{ }^{\circ} \mathrm{C}$ & $292-322{ }^{\circ} \mathrm{C}$ & & 82.12 \\
\hline & HWTHH & - & $369{ }^{\circ} \mathrm{C}$ & - & 80.70 \\
\hline \multirow[t]{4}{*}{ Chemical (air atmosphere) } & RHH & $312{ }^{\circ} \mathrm{C}$ & $395{ }^{\circ} \mathrm{C}$ & $437{ }^{\circ} \mathrm{C}$ & 94.90 \\
\hline & ATHH & - & $352{ }^{\circ} \mathrm{C}$ & $342-382{ }^{\circ} \mathrm{C}$ & 95.99 \\
\hline & ETHH & $300{ }^{\circ} \mathrm{C}$ & $300-362{ }^{\circ} \mathrm{C}$ & $362-512{ }^{\circ} \mathrm{C}$ & 95.55 \\
\hline & CTHH & $328^{\circ} \mathrm{C}$ & $343{ }^{\circ} \mathrm{C}$ & $551{ }^{\circ} \mathrm{C}$ & 93.69 \\
\hline \multirow[t]{3}{*}{ Physico-chemical (air atmosphere) } & UWTHH & - & $222-375^{\circ} \mathrm{C}$ & - & 94.58 \\
\hline & UATHH & $207-336^{\circ} \mathrm{C}$ & $336-413{ }^{\circ} \mathrm{C}$ & $413-438^{\circ} \mathrm{C}$ & 93.50 \\
\hline & HWTHH & $229-371{ }^{\circ} \mathrm{C}$ & $371-550{ }^{\circ} \mathrm{C}$ & 94.22 & \\
\hline
\end{tabular}

hemicelluloses and cellulose given in Table 4 (77.3\%). The found difference relates to the mass loss due a partial decomposition of lignin. Total mass loss due thermal degradation of hemp hurds constituents in air is $94.9 \%$, what is relatively in good accordance with chemical analysis of organic materials $(96.2 \%)$.

As it is evident from Table 4, lignin degradation proceeds gradually over a broader temperature range than two components of holocellulose. In the first and second temperature range, lignin decomposition occurs simultaneously with hemicelluloses and cellulose degradation. Third exothermic peak observed corresponding to only lignin is recorded on DSC curves at $437{ }^{\circ} \mathrm{C}$ (air) and in range of $311-384{ }^{\circ} \mathrm{C}$ (nitrogen). The thermal analysis of extracted lignin from hemp fibers showed that decomposition lignin took place in a broad temperature range $\left(150-450{ }^{\circ} \mathrm{C}\right)$ [27]. Lignin decomposition is accompanied by formation of aromatic hydrocarbons, hydroxyphenolics, guaiacyl and syringyl compounds [46]. The study [44] revealed that decomposition temperature range of lignin in hemp fibers is $280-500{ }^{\circ} \mathrm{C}$. The corresponding mass loss was about $40.4 \%$. According to [26], the DTA curve (air) of natural hemp fibers above $380{ }^{\circ} \mathrm{C}$ presents exothermic peaks at 395 and $427{ }^{\circ} \mathrm{C}$ and the mass loss (\%) is about $27 \%$. These peaks are attributed to the oxidative decomposition of the charred residue. No peaks in nitrogen atmosphere were seen. This result confirms that these peaks are produced by the residue loss in air. Decomposition of natural hemp fibers under air was complete and proceeded at a lower temperature than under inert atmosphere. According to [18], the decomposition of hemp fibers in nitrogen atmosphere is due to the presence of free radicals.

\section{Chemically treated hemp hurds}

DSC and TG/DTG curves (nitrogen and air atmosphere) of chemically treated hemp hurds (ATHH, CTHH and ETHH) in water solutions of $\mathrm{NaOH}, \mathrm{Ca}(\mathrm{OH})_{2}$ and EDTA are shown in Figs. 1b-d and 2b-d. As seen from comparison of the DSC measurements in Figs. 3 and 4, the curves for chemically modified hemp hurds samples have a different course in dependence on the used agent's nature compared to the referential sample (RHH). In the case of all chemically treated samples, the onset of temperature degradation of the hemp hurds components is observed at higher temperatures in comparison with the RHH (Table 4). It means that higher temperature is needed to remove hemp hurds constituents. As a result, thermal stability of the chemically treated hemp hurds samples was higher in comparison with the untreated sample.

As above mentioned, the presence of an endothermic peak in the temperature range of $40-150{ }^{\circ} \mathrm{C}$ corresponds to a mass loss of the adsorbed moisture of about 1.8-2.2\% compared with 3-4\% determined for RHH sample (air and nitrogen atmosphere). This difference could indicate surface cleaning of hemp samples from fats, waxes and others.

The endothermic peak observed in a $220-370{ }^{\circ} \mathrm{C}$ temperature range in air corresponds to the simultaneous decomposition of hemicelluloses, cellulose and lignin with a large mass loss about $60 \%$ (Fig. 4b-d). This probably indicates that the higher amount of cellulose was degraded at lower temperature in comparison with RHH. As shown in [22], cellulose percentage increased after all chemical treatments. This was also confirmed by the increase in the cellulose crystallinity index, determined by X-ray diffraction as well as by FTIR measurements. 
Fig. 3 Comparison of thermal decomposition of chemically treated samples measured under nitrogen atmosphere ( $\mathrm{RHH}-$ reference hemp hurds; ATHH$\mathrm{NaOH}$ treated; $\mathrm{CTHH}-$ $\mathrm{Ca}(\mathrm{OH})_{2}$ treated; $\mathrm{ETHH}-$ EDTA treated)

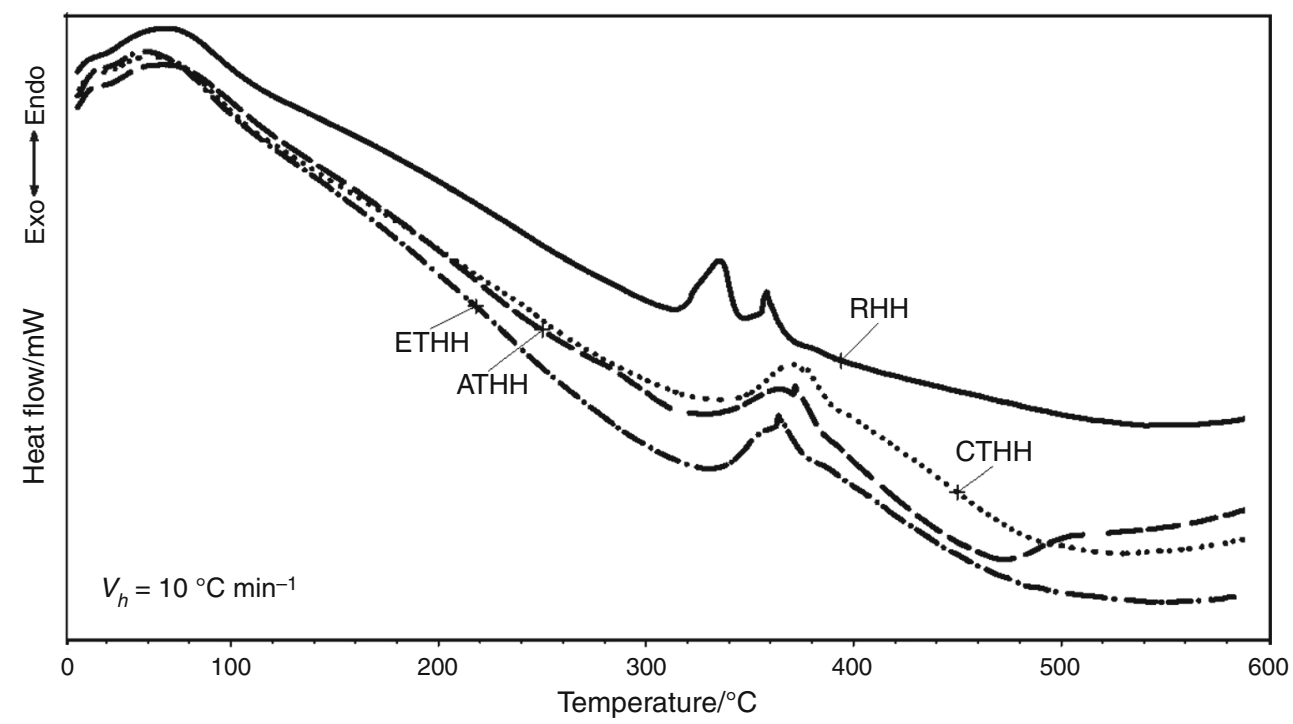

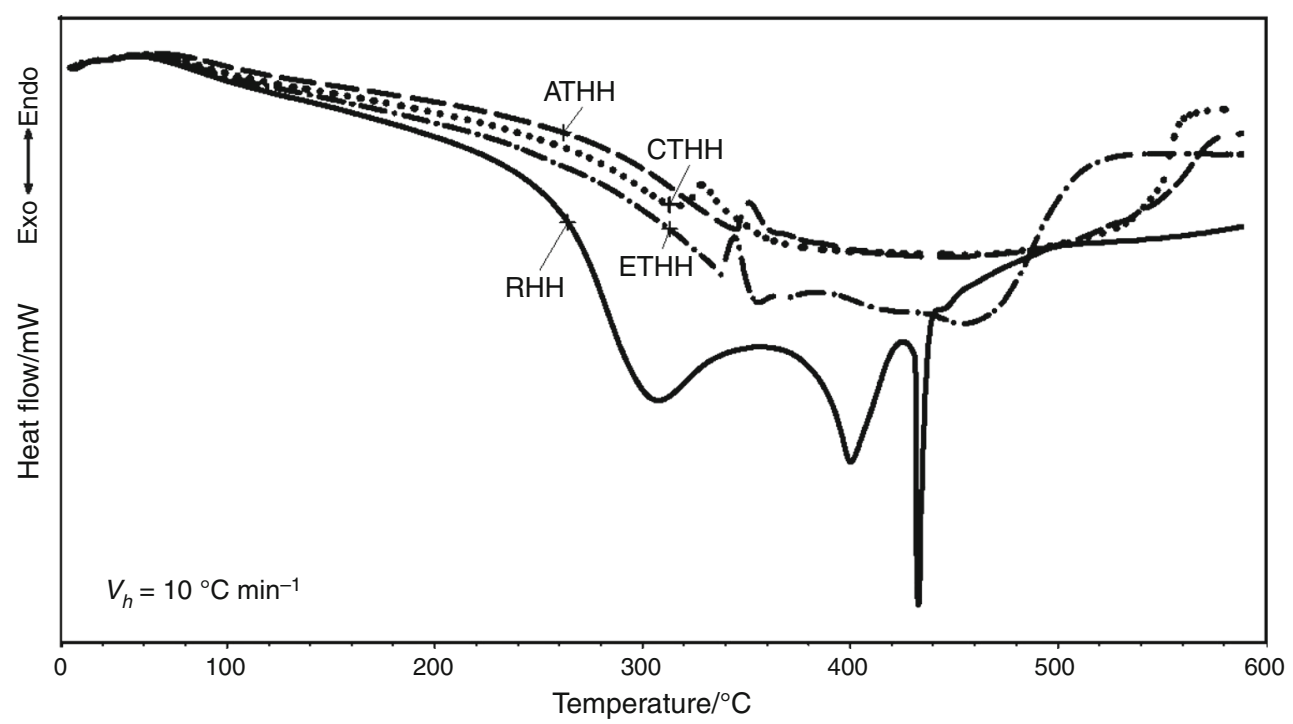

Fig. 4 Comparison of thermal decomposition of chemically treated samples measured in air (RHH-reference hemp hurds; ATHH-NaOH treated; $\mathrm{CTHH}-\mathrm{Ca}(\mathrm{OH})_{2}$ treated; ETHH-EDTA treated)

However, the interpretation of DSC measurements of cellulosic materials is complicated by the decomposition of lignin residues over the whole temperature range $\left(280-500{ }^{\circ} \mathrm{C}\right)$. In all cases of treatment of hemp hurds, degradation of cellulose is difficult to disentangle on the DDSC (derived differential scanning calorimetry) curves because of the peaks overlapping. We were able to determine only the top peak but not its whole course. These findings are in accordance with data from the literature [47]. Top of peaks of cellulose decomposition was recorded about $364-378{ }^{\circ} \mathrm{C}$ (nitrogen) and $343-352{ }^{\circ} \mathrm{C}$ (air).

Moreover, the shoulder of the DSC peak at (air) about $300-335{ }^{\circ} \mathrm{C}$ observed on Fig. $4 \mathrm{a}$ and attributed to the depolymerization of hemicelluloses and pectin, disappeared after treatment by $\mathrm{NaOH}$ (ATHH) and its intensity is reduced after EDTA and $\mathrm{Ca}(\mathrm{OH})_{2}$ modification (ETHH and CTHH). On the other hand, in nitrogen atmosphere, two exothermic peaks (Fig. 3b-d) in the range of $300-420{ }^{\circ} \mathrm{C}$ on the DSC curves of chemically treated samples are recorded. It is attributed to the pectin and hemicelluloses depolymerization as well as cellulose and lignin decomposition. The corresponding mass loss is $44-63 \%$ in dependence on used modification agents (Table 4). This fact confirms the removal of a large part of hemicelluloses and pectin by chemical treatment, especially by alkaline treatment as described in previous part. The decrease in hemicelluloses amount due to chemical treatment was also confirmed by FTIR spectroscopy [22]. 


\section{Physico-chemically treated hemp hurds}

The DSC and TG/DTG curves of three physico-chemically treated (ultrasonification in water and $\mathrm{NaOH}$ solution; hot water treatment) hemp hurds under experimental conditions in air and nitrogen atmosphere are shown in Figs. 1e-g and $2 \mathrm{e}-\mathrm{g}$.

As shown in Table 4 and Figs. 5 and 6, degradation processes of hemicelluloses and decomposition of cellulose and lignin begins at the lower temperatures under nitrogen atmosphere in comparison with processes under air atmosphere. DTG curves of UWTHH sample (Figs. 1f, 2f) show that degradation processes took place simultaneously in temperature ranges $207-382$ and $222-375^{\circ} \mathrm{C}$ under nitrogen and air atmospheres, respectively. The found mass losses during the heating stage were similar (64.3 and $62.6 \%$ under nitrogen and air atmosphere, respectively). It seems that degradation processes run together for samples treated by ultrasonification in water. As shown in Table 4 and Fig. 4, degradation processes in nitrogen atmosphere were in progress at $178-347^{\circ} \mathrm{C}$ for UATHH sample with lower mass loss $51.2 \%$ in comparison with UWTHH. In this case, two peaks were observed in the range of 292-322 ${ }^{\circ} \mathrm{C}$, belonging to degradation of hemicelluloses and cellulose with lignin. In air atmosphere, three peaks were recorded (Fig. 6). The first peak at $207-336{ }^{\circ} \mathrm{C}$ (mass loss was $55.4 \%$ ) is connected with hemicelluloses depolymerization and simultaneously begins degradation of lignin. The second peak at $336-413{ }^{\circ} \mathrm{C}$ (mass loss was $15.4 \%$ ) corresponds to cellulose decomposition and continuous degradation of lignin. The last decomposition is finished at $438{ }^{\circ} \mathrm{C}$. Based on DTG curves (Fig. 2e), peak observed over $483{ }^{\circ} \mathrm{C}$ is attributed to the oxidative decomposition of the charred residue. As in the case of chemically modified samples, this peak is not detected for UWTHH and UATHH in nitrogen atmosphere.

Hemp hurds treated by boiling in hot water (HWTHH) and thermally analyzed in nitrogen atmosphere exhibited overlapping depolymerization processes of aforesaid compounds. Thermal degradation actions started at 223-384 ${ }^{\circ} \mathrm{C}$, the mass loss was $66 \%$. In air atmosphere, it occurs in the temperature range of $229-371{ }^{\circ} \mathrm{C}$ with mass loss of $63.5 \%$. Next mass loss $(27.1 \%)$ was recorded in the range of $371-550{ }^{\circ} \mathrm{C}$. These two mass losses are connected with depolymerization of hemicelluloses and pectin and decomposition of cellulose and degradation of lignin as well as with oxidative decomposition of the charred residue, probably.

As the result of thermal analysis, different behavior of hemp hurds samples in dependence on used treatment method. Higher thermal stability of modified samples compared to reference sample indicates their improved hydrophobic nature and higher adhesion possibility with matrix particles.

\section{Relationship of thermally released water content and degree of cellulose polymerization}

Cellulose as semi crystalline polysaccharide is the main hemp hurds component covered by hemicelluloses and lignin constituents. Hemicelluloses and lignin polymers are fully amorphous. Degree of polymerization (DP) is one of the main factors playing role in the aging of cellulose fibers. Processes of aging are associated with the degradation of cellulose macromolecules, increasing the proportion of low molecular mass fraction and decreasing DP. As previously reported treatment process leads to removal of a certain amount of hemicelluloses and lignin,
Fig. 5 Comparison of thermal decomposition of physicochemically treated samples measured under nitrogen atmosphere (RHH-reference hemp hurds; HWTHH-hot water boiled; UWTHHultrasound treated in water; UATHH - ultrasound treated in $\mathrm{NaOH}$

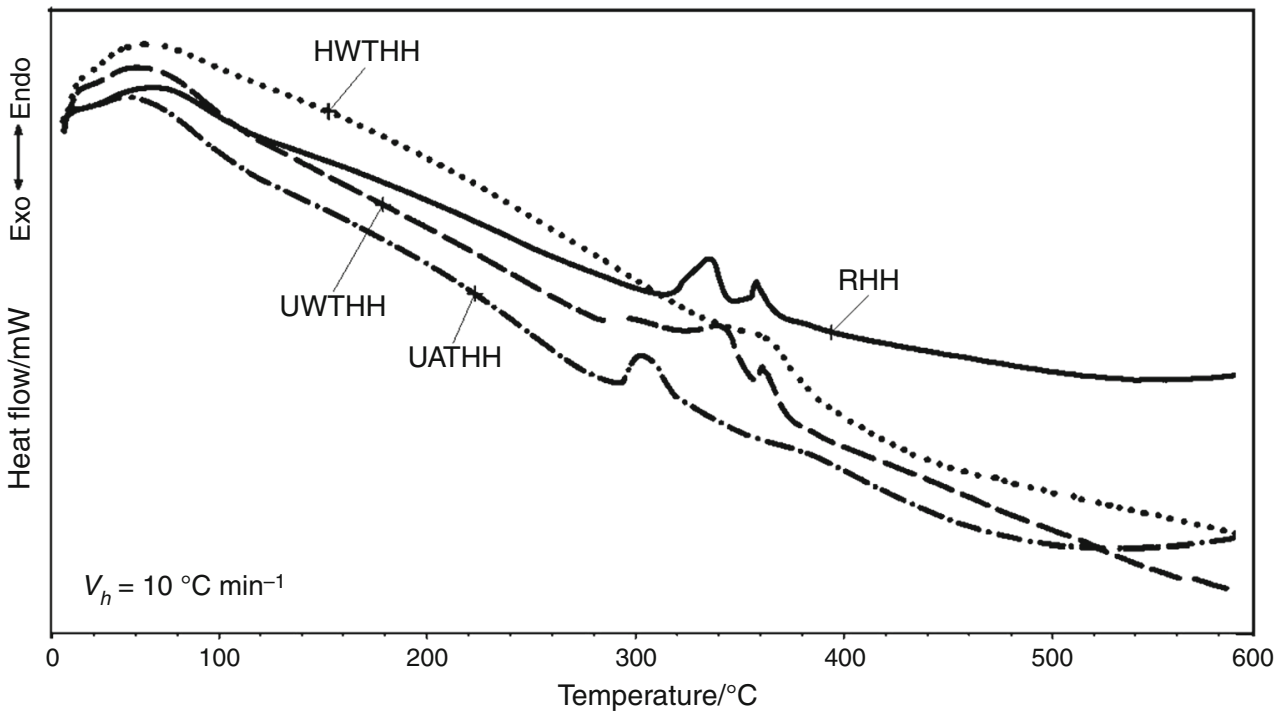


Fig. 6 Comparison of thermal decomposition of physicochemically treated samples measured in air (RHHreference hemp hurds; HWTHH - hot water boiled; UWTHH—ultrasound treated in water; UATHH-ultrasound treated in $\mathrm{NaOH}$ )

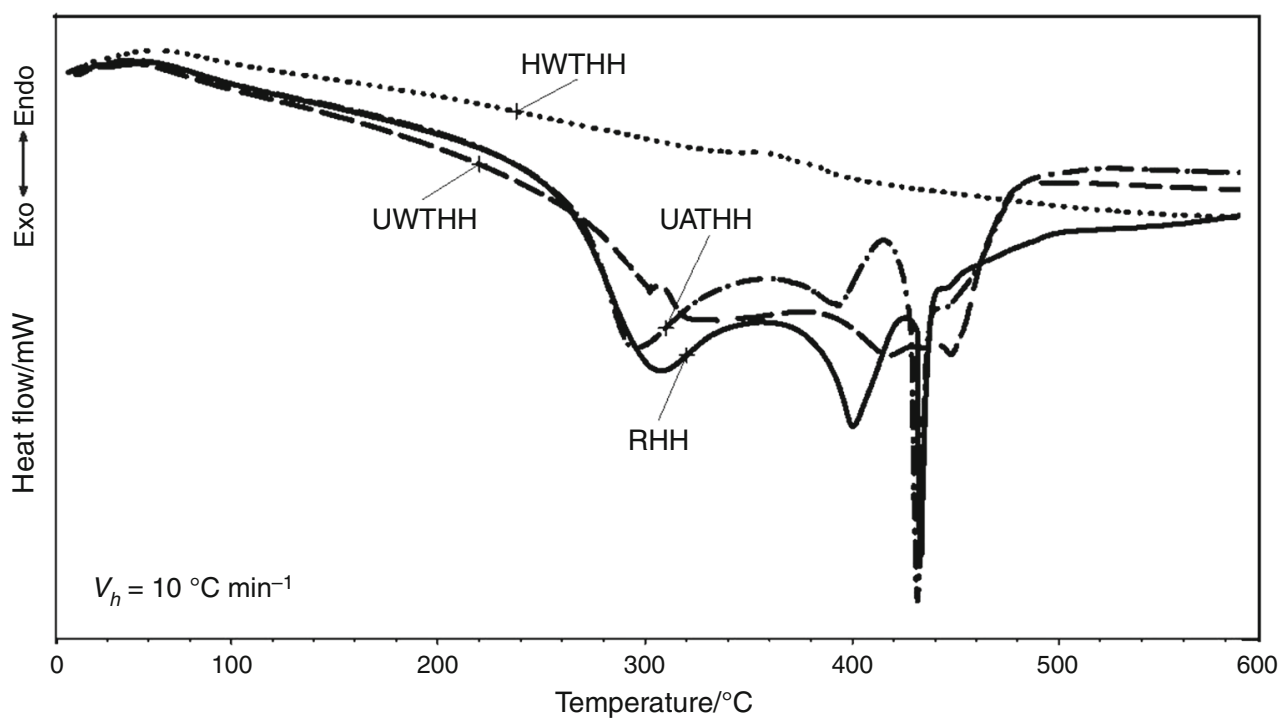

defibrillation of bundles of microfibrils and breaking the cellulose chains into shorter fragments. As a result, larger number of potential reactive sites of cellulose is available. These metastable centers present in cellulose can be combined with water molecules. Hydroxyl groups present in the residual portion of amorphous hemp hurds components such as lignin and hemicelluloses are also capable to react with water molecules from the atmosphere. Based on this hypothesis we can assume, the lower is a degree of cellulose polymerization, the higher is water content in treated hemp hurds samples. As the TG records of hemp hurds samples in nitrogen and air atmosphere showed, processes of mass loss reflect the thermo-oxidative decomposition of lignocellulosic material. The decrease in the mass of the all studied hemp hurds samples (reference and treated samples) from 8.0 to $2.8 \%$ (air) and from 5.8 to $2.5 \%$ (nitrogen) up to $120{ }^{\circ} \mathrm{C}$ corresponds to the moisture evaporation. These results of mass loss were used for correlation with degree of cellulose polymerization values. Correlation between experimentally determined parameters of the moisture content released under nitrogen and air atmosphere and DP of cellulose in hemp hurds was fitted by several functions but as shown in Figs. 7 and 8, a linear dependence was found to be the most suitable. Each point represents the average value of four measurement data from each parameter. As the measured values variability of the arithmetic average have a small relative standard deviation in the range of $2.6-4.8 \%$, the average values of monitored parameters can be considered as representative ones in the dependence. Such a set of 28 values contains sufficient data for correct statistical analysis of the variance of measured data around the regression line.

The values of calculated correlation coefficients 0.796 (nitrogen) and 0.922 (air) are higher than the critical value of the correlation coefficient $(0.754)$ for a set of measured values at elected significance level $p=0.05$. The degree of correlation closeness is high because correlation coefficients $R$ are in the interval $0.7<R<0.9$. The existence of the linear relationship between variables such as water content and degree of cellulose polymerization at given significance level can be regarded as proven. This dependence confirms that higher moisture amount is released from fragments of polymeric cellulose with shorter chain. This phenomenon can be explained by higher number of active centers/unsaturated bonds at surface due to disruption of hydrogen bonds in cellulose structure after treatment procedure and greater ability to bind the water molecules from the atmosphere.

As reported in [48], an exponential dependence between moisture content and degree of cellulose polymerization in cotton samples was identified. In the contrast to the treated hemp hurds samples, the higher water content in washed cotton samples is associated with higher DP of cellulose.

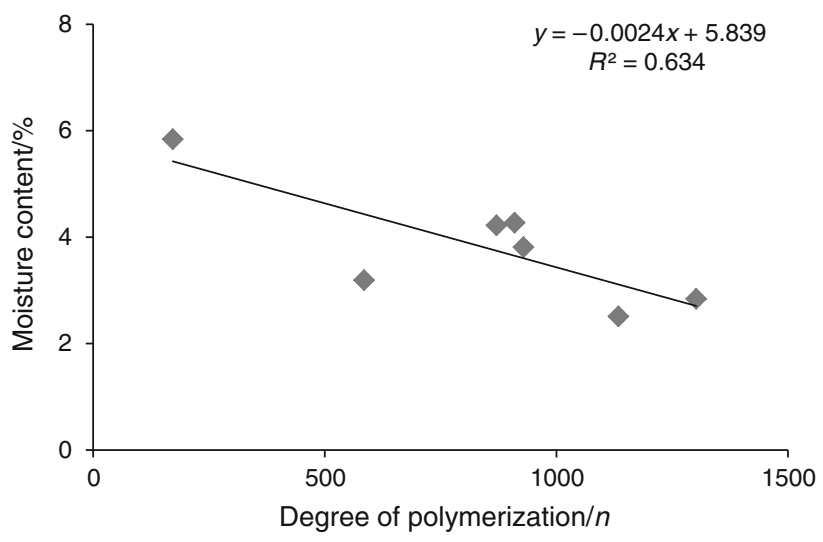

Fig. 7 Relationship between moisture content released during thermal analysis of hemp hurds samples in nitrogen atmosphere and degree of cellulose polymerization 


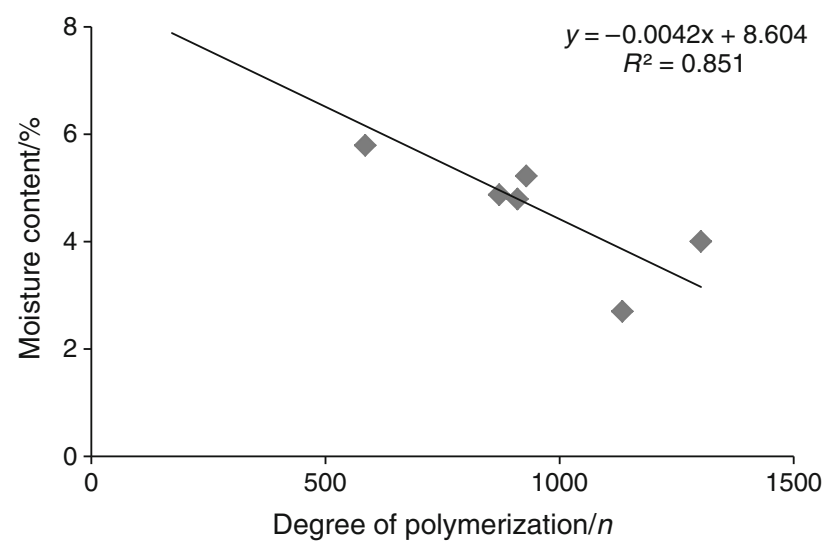

Fig. 8 Relationship between moisture content released during thermal analysis of hemp hurds samples in air atmosphere and degree of cellulose polymerization

\section{Conclusions}

In the present work, the effects of different procedures of treatment on the thermal stability of hemp hurds were investigated. The results obtained by the chemical analysis of hemp hurds and TG/DSC measurements seem be some contribution to the understanding the differences in behavior of hemp hurds samples before and after their treatment. They are summarized, as follows:

1. Surface treatment influences the chemical composition and complex structure of hemp hurds. Based on contents of non-cellulosic compounds of treated samples, alkaline modification of hemp hurds in $\mathrm{NaOH}$ and combination of ultrasonification in alkaline solution seem to be the most effective procedures in removal of hemicelluloses and lignin.

2. The different thermal behavior was found for the hurds samples under nitrogen and air heating. TG records of hemp hurds samples showed, several-stage process of mass loss, reflecting the thermo-oxidative decomposition of lignocellulosic material under air whereas twostage process under nitrogen. Heating of cellulosic materials under air atmosphere led to oxidation of hydroxyl groups in cellulose molecules and consequently to an increase in carbonyl groups number and carboxylate compounds formation. Under nitrogen, the depolymerization reactions dominated.

3. The decomposition processes depend also on the chemical properties of hemp hurds influenced by treatment procedure. Treatment by different ways led to the shifts of decomposition temperatures to higher temperatures. As a result, higher thermal stability of modified hemp hurds samples due to removal of the binding constituents was observed in dependence on used treatment method. Higher thermal stability of modified samples compared to reference sample indicates their improved hydrophobic nature and higher adhesion possibility with matrix particles. Experimental results suggest that hemicelluloses are the most reactive constituent; it is more easily thermally degraded than the other hemp hurds componentscellulose and lignin.

4. The existence of a linear relationship between released water content and degree of cellulose polymerization at significant level was found. Higher moisture amount is released from fragments of polymeric cellulose with shorter chain. This phenomenon can be explained by higher number active centers/unsaturated bonds at surface due to disruption of hydrogen bonds in cellulose structure after treatment procedure and the greater ability to bind the water molecules.

Acknowledgements The authors are grateful to the Slovak Grant Agency for financial support of the project VEGA 1/0277/15 and to the Agency of the Ministry of Education, Science, Research and Sport of the Slovak Republic for the Structural Funds of EU for supporting the project NFP 26220120037.

Open Access This article is distributed under the terms of the Creative Commons Attribution 4.0 International License (http://creative commons.org/licenses/by/4.0/), which permits unrestricted use, distribution, and reproduction in any medium, provided you give appropriate credit to the original author(s) and the source, provide a link to the Creative Commons license, and indicate if changes were made.

\section{References}

1. Mangino E, Pitarresi G. The future use of structural composite materials in the automotive industry. Int $\mathrm{J}$ Vehicle Des. 2007;44:211-32.

2. Elfordy S, Lucas F, Tancret F, Scudeller Y, Goudet L. Mechanical and thermal properties of lime and hemp concrete ("hempcrete") manufactured by a projection process. Constr Build Mater. 2008;22:2116-23.

3. Arnaud L, Gourlay E. Experimental study of parameters influencing mechanical properties of hemp concrete. Constr Build Mater. 2012;28:50-6.

4. Kim SC, Klempner D, Frisch KC, Frisch HL. Polyurethane interpenetrating polymer networks. V. Engineering properties of polyurethane-poly(methyl methacrylate) IPN's. J Appl Polym Sci. 1977;21:1289-95.

5. Yao F, Wu Q, Lei Y, Guo W, Xu Y. Thermal decomposition kinetics of natural fibers: activation energy with dynamic thermogravimetric analysis. Polym Degrad Stab. 2008;93:90-8.

6. Siti Alwani M, Abdul Khalil HPS, Sulaiman O, Nazrul Islam M, Dungani R. An approach to using agricultural waste fibres in biocomposites application: thermogravimetric analysis and activation energy study. BioRes. 2014;9:218-30.

7. Cruz G, Crnkovic PM. Investigation into kinetic behaviour of biomass combustion under $\mathrm{N}_{2} / \mathrm{O}_{2}$ and $\mathrm{CO}_{2} / \mathrm{O}_{2}$ atmospheres. J Therm Anal Calorim. 2016;123:1003-11.

8. Braga RM, Costa TR, Freitas JCO, Barros JMF, Melo DMA, Melo MAF. Pyrolysis kinetics of elephant grass pretreated biomass. J Therm Anal Calorim. 2014;117:1341-8. 
9. Poletto M, Ornaghi HL Jr, Zattera A. Thermal decomposition of natural fibres: kinetics and degradation mechanisms, Chapter 21. In: Tiwari A, Raj B, editors. Reactions and mechanisms in thermal analysis of advanced materials. Hoboken, NJ: Wiley; 2015. p. 515-46.

10. Zhang ZH, Zhao ZBK. Microwave-assisted conversion of lignocellulosic biomass into furans in ionic liquid. Bioresour Technol. 2010;101:1111-4.

11. Carus M, Dammer L. Food or non-food: which agricultural feedstocks are best for industrial uses? Ind Biotechnol. 2013;9:171-6.

12. Paze A, Rizhikovs J, Puke M, Tupciauskus R, Andzs M, Meile K. Changes in the polysacharide complex of lignocellulose after catalytic hydrothermal pre-treatment process of Hemp (Cannabis Sativa L.) shives. In: Proceedings of 23rd European Biomass Conference and Exhibition. Vienna, Austria. 1-4 June 2015. pp. 1063-1069.

13. CarusM, Karst S, Kauffmann A, Hobson J, Bertucelli S. European Hemp Industry: Cultivation, processing and applications for fibres, shivs and seeds. EIHA. 2013. 1-9. http://www.eiha.org. Accessed 22 Apr 2016.

14. Karus M. European hemp industry: cultivation, processing, and product lines. EIHA. 2002. http://www.eiha.org. Accessed 15 Jan 2016.

15. Vignon MR, Dupeyre D, Garcia-Jaldon C. Morphological characterization of steam-explosed hemp fibres and their utilization in polypropylene-based composites. Bioresour Technol. 1996;58:203-15.

16. Liu X, Bai X, Zhang Z. Thermal stability of the hemp fibers and fabrics. Adv Mater Res. 2012;466-467:152-6.

17. Bilbao R, Millera A, Arauzo J. Thermal decomposition of lignocellulosic material-influnce otf the chemical composition. Thermichim Acta. 1989;143:149-59.

18. Quajai S, Shanks RA. Composition, structure and thermal degradation of hemp cellulose after chemical treatments. Polym Degrad Stab. 2005;89:327-35.

19. Oza S, Ning H, Ferguson I, Lu N. Effect of surface treatment on thermal stability of the hemp-PLA composites: correlation of activation energy with thermal degradation. Compos B. 2014;67:227-32.

20. Bledzki AK, Gassan J. Composites reinforced with cellulose based fibres. Prog Polym Sci. 1996;24:221-74.

21. Yang P, Kokot S. Thermal analysis of different cellulosic fabrics. J Appl Polym Sci. 1996;60:1137-46.

22. Stevulova N, Cigasova J, Estokova A, Terpakova E, Geffert A, Kacik F, Singovszka E, Holub M. Properties characterization of chemically modified hemp hurds. Material. 2014;7:8131-50.

23. Shazad A. A study in physical and mechanical properties of hemp fibres. Adv Mater Sci Eng. 2013;. doi:10.1155/2013/325085.

24. Prasad BM, Sain MM, Roy DN. Properties of ball milled thermaly treated hemp fibers in an inert atmosphere for potential composite reinforcement. J Mater Sci. 2005;40:4271-8.

25. Sebestyén Z, May Z, Réczey K, Jakab E. The effect of alkaline pretreatment on the thermal decomposition of hemp. J Therm Anal Calorim. 2011;105:1061-9.

26. Rachini A, Le Troedec M, Peyratout C, Smith A. Comparison of the thermal degradation of natural, alkali-treated and silanetreated hemp fibres under air and inert atmosphere. J Appl Polym Sci. 2009;112:226-34.

27. Kabir MM, Wang H, Lau KT, Cardona F. Effects of chemical treatments on hemp fibre structure. Appl Surf Sci. 2013;276:13-23.

28. Le Troëdec M, Sedan D, Peyratout C, Bonnet JP, Smith A, Guinebretiere R, Gloaguen V, Krausz P. Influence of chemical treatments on adhesion properties of hemp fibres. Compos A. 2008;39:514-22.
29. Wise LE, Maxine M, D'Addieco AA. Chlorite holocellulose, its fractionation and bearing on summative wood analysis and on studies on the hemicelluloses. Pap Trade J. 1946;122:35-43.

30. Seifert VK. Über ein neues Verfahren zur Schnellbestimmung der Rein-Cellulose. Papier. 1956;10:301-6.

31. Sluiter A, Hames B, Ruiz R, Scarlata C, Sluiter J, Templeton D, Crocker D. Determination of structural carbohydrates and lignin in biomass; Technical Report NREL/TP-510-42618. National Renewable Energy Laboratory: Golden. CO. USA. 2010.

32. Sluiter A, Hames B, Ruiz R, Scarlata C, Sluiter J, Templeton D. Determination of ash in biomass; Technical Report NREL/TP510-42622. National Renewable Energy Laboratory: Golden. CO. USA. 2008

33. Kacik F, Kacikova D, Jablonsky M, Katuscak S. Cellulose degradation in new-sprint paper ageing. Polym Degrad Stab. 2009;94:1509-14.

34. Renouard S, Hano C, Doussot J, Blondeau JP, Lainé E. Characterization of ultrasonic impact on coir, flax and hemp fibers. Mater Lett. 2014;129:137-41.

35. Strobin G, Ciechanska D, Wawro D, Boryniec S, Struszczyk H, Sobczak S. Gel permeation chromatography of chemically, irradiated and enzymatically treated cellulosics. Fibres Text East Eur. 2003;11:62-5.

36. Emsley AM, Ali M, Heywood RJ. A size exclusion chromatography study of cellulose degradation. Polymer. 2000;41:8513-21.

37. Lewin M. Handbook of fiber chemistry. 3rd ed. Boca Raton: Taylor and Francis Group; 2007.

38. Suslick KS. Sonochemistry. Science. 1990;247:1439-45.

39. Gonçalves I, Herrero-Yniesta V, Perales Arce I, Escrigas Castañeda M, Gonçalves I, Herrero-Yniesta V, Perales Arce I, Escrigas Castañeda M, Cavaco-Paulo A, Silva C. Ultrasonic pilot-scale reactor for enzymatic bleaching of cotton fabrics. Ultrason Sonochem. 2014;21:1535-43.

40. Bourgois J, Bartholin MC, Guyonett R. Thermal treatment of wood: analysis of obtained products wood. Wood Sci Technol. 1989;23:303-10.

41. Yildiz S, Gezer ED, Yildiz UC. Mechanical and chemical behaviour of spruce wood modified by heat. Build Environ. 2006;41:1762-6.

42. Kacik F, Smira P, Kacikova D, Velkova V, Nasswettrova A, Vacek V. Chemical alternations of pine wood saccharides during heat sterilisation. Carbohydr Polym. 2015;117:681-6.

43. Einhorn-Stoll U, Kunzek K, Dongowski G. Thermal analysis of chemically and mechanically modified pectins. Food Hydrocolloid. 2007;21:1101-12.

44. Kifani-Sahban F, Belkbir L, Zoulalian A. Etude de la pyrolyse lente de l'Eucalyptus marocain par analyse thermique. Thermochim Acta. 1996;284:341-9.

45. Lopez-Velazques MA, Santes V, Balmaseda I, Torres-Garcia E. Pyrolysis of orange waste: a thermo-kinetic study. J Anal Appl Pyrolysis. 2013;99:170-7.

46. Brebu M, Vasile C. Thermal degradation of lignin-a review. Cell Chem Technol. 2010;44:353-63.

47. Lu N, Shubhashini O. Thermal stability and thermo-mechanical properties of hemp-high density polyethylene composites: effect of two different chemical modifications. Compos B. 2013;44:484-90.

48. Sperova M, Nasadil M, Prusova A, Kucerik J. A hint on the correlation between cellulose fibers polymerization degree and their thermal and thermo-oxidative degradation. J Therm Anal Calorim. 2012;110:71-6. 